\title{
Using interprofessional dementia learning opportunities to prepare the future healthcare workforce: Findings from a pilot study
}

Mrs Elizabeth Davison ${ }^{\text {a }}$ Dr Sarah Housden ${ }^{\mathrm{b}}$; Prof. Susanne Lindqvist ${ }^{\mathrm{c}}$

${ }^{a}$ Centre for Interprofessional Practice, University of East Anglia, Norwich, Norfolk, UK; ${ }^{b}$ School of Health Sciences, University of East Anglia, Norwich, Norfolk, UK; 'C Centre for Interprofessional Practice, University of East Anglia, Norwich, Norfolk, UK.

\section{Corresponding author:}

Professor Susanne Lindqvist $\bowtie \underline{\text { s.lindqvist@uea.ac.uk }}$

$\equiv$ Centre for Interprofessional Practice, University of East Anglia, Norwich Research Park, Norwich, NR4 7JT, UK. 酒 01603591274.

Keywords: Dementia; aging society; qualification; communication difficulty; interprofessional learning; online.

\section{Abstract}

Nearly 50 million people worldwide are living with dementia. Communication difficulties linked to this illness demand that all healthcare professionals are prepared to meet the needs of this group of service users. In response to this, the United Kingdom government is calling for professionals to acquire a basic (Tier 1) Dementia Awareness (DA) qualification. As healthcare students need to engage in interprofessional learning (IPL), this report describes the development, implementation and evaluation of an initiative to link the DA qualification to an existing IPL module delivered to first-year healthcare students (IPL1). A DA learning package was developed by a group of educators from a range of professions to ensure an interprofessional focus. It comprised of a set of practical exercises that students completed during and after IPL1. Sixty students evaluated the DA learning package by completing a postintervention survey. 57 students rated it, or very helpful in enhancing their knowledge of how to care for a person with dementia, while 3 students rated it average. Two themes emerged from open-ended questions, which highlighted the importance of: i) learning to work 
together; and ii) blended learning. Students also suggested some changes for the full roll out, such as moving the Dementia Friends component into IPL1. This is an innovative approach that can be used to meet the challenges linked with the large-scale preparation of our future workforce and to ensure purposeful IPL.

\section{Introduction}

While Alzheimer's Disease International (2016) estimated that 47 million people live with dementia, Alushi, Hammond \& Wood (2015) argued that our current workforce is inadequately prepared to deal with this growing group of patients. To address this, HEE (Health Education England), a government organisation responsible for workforce education and training, published a mandate in 2015 stating that all National Health Service (NHS) staff should acquire a basic Tier 1 Dementia Awareness (DA) qualification. This DA qualification is linked with a set of Learning Outcomes (LOs) that provide professionals with the knowledge, skills, attitudes, values and behaviours needed to care for people with dementia.

Educational staff at the University of East Anglia (UEA) in the United Kingdom (UK) decided that all their healthcare students should be given the opportunity to complete the Tier $1 \mathrm{DA}$ qualification during their course. Since all healthcare students UEA come together during an interprofessional learning (IPL) module in their first year, it was agreed that this would serve as a platform for this initiative.

Despite limited evidence to suggest that IPL has a positive impact on dementia care (Jackson et al. 2016), some small studies have been presented in the literature. For example, Cartwright et al. (2015) trialed an online study for 125 students that aimed to promote 
collaborative working when caring for people with dementia. Despite some positive findings, the authors highlighted a number of challenges linked to making it compulsory, and the need for students to also interact face-to-face. Balzer (2016) developed an existing interprofessional course to improve students' competencies in evidence-based dementia care. Findings confirmed the relevance of an interprofessional approach, but again highlighted logistical challenges to large-scale roll out.

In order to provide a solution to the commonly reported difficulties linked with engaging a large number of students in purposeful IPL activities, this short report describes the development, implementation and evaluation of a pilot that links a Tier 1 DA qualification to an existing IPL module delivered to 812 first-year students (IPL1).

\section{Background}

A group of 14 educators from medicine, nursing, paramedic science, pharmacy, midwifery, operating department practice, speech and language therapy, physiotherapy and occupational therapy, developed a learning package for students to gain the DA qualification. It included: a one-hour Dementia Friends ${ }^{1}$ session that could be completed either face-to-face in an interprofessional group, or online; a video on family members sharing experiences of dementia; a reflective student workbook; and a multiple-choice-questionnaire (MCQ) consisting of 10 questions that related to the IPL1 session and the learning package. Students were required to answer eight out of the 10 questions in the $\mathrm{MCQ}$ correctly in order to obtain the Tier 1 DA qualification. 


\section{Methods}

\section{Study Design}

A post-intervention survey was developed using Survey Monkey comprising 20 questions that generated numerical data and open-ended text-based responses.

\section{Sample}

All 812 students of the $2016-2017$ cohort who had successfully completed IPL1 were invited to complete and evaluate the DA learning package. Table 1 provides a breakdown of the 60 student who volunteered to take part this pilot.

Table 1. Breakdown of student professions who volunteered to complete and evaluate the experience of completing IPL1 and the DA learning package

\begin{tabular}{lc}
\hline Course & Total \\
Medicine & 15 \\
Nursing & 20 \\
Speech and Language Therapy & 6 \\
Occupational Therapy & 6 \\
Physiotherapy & 7 \\
Pharmacy & 5 \\
Paramedic Science & 1
\end{tabular}

Data collection

Question one asked students to confirm their course. Question two assessed students' overall rating of this initiative in enhancing their knowledge of caring for a person with dementia using a 5-point Likert scale ( 5 = very helpful; 4 = helpful; $3=$ average; 2 = poor; $1=$ very poor). The remaining 18 open-ended questions assessed students' perceptions of this experience in relation to how they will care for a person with dementia. 


\section{Data analysis}

Descriptive statistics was applied to the numeric data, and data from open-ended text-based responses were subjected to thematic analysis to inform further development.

\section{Ethical considerations}

Ethical approval was not necessary, as this was a curricular development. Participants were assured of strict anonymity and could opt out at any time.

\section{Results}

57 (95\%) of the respondents scored the opportunity to gain the Tier 1 DA qualification in this way as helpful, or very helpful, in enhancing their knowledge of how to care for a person with dementia. 3 students (5\%) scored it as average.

Table 2. Summary of students' evaluation of the learning experience showing the number of students scoring it as average, helpful and very helpful, respectively.

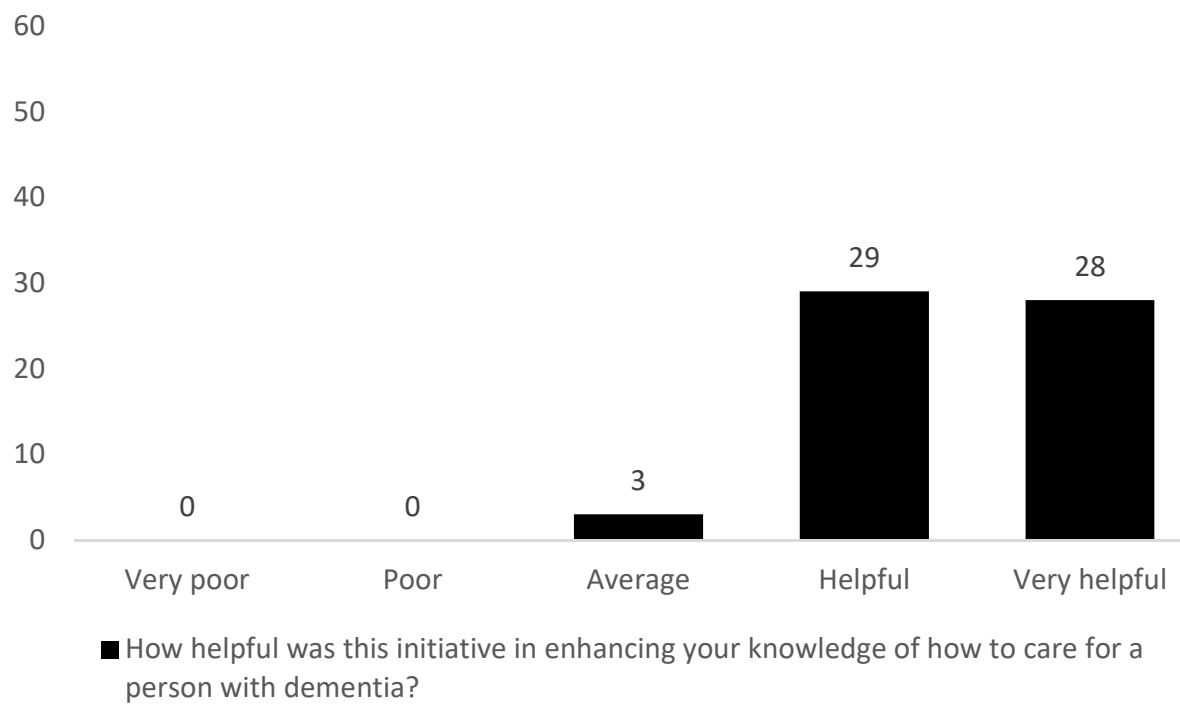

Helpful

28

Very helpful

- How helpful was this initiative in enhancing your knowledge of how to care for a person with dementia? 
Data from the open-ended comments from the survey highlighted two key themes:

1) Learning to work together

2) Blended learning

Students highlighted clear benefits from learning together during IPL1, where they gained a deeper insight into the challenges faced by people with communication difficulties and together explored ways to overcome them:

[It was] useful to work in an interprofessional team as practice for the future, helpful exercise in promoting communication. (Respondent 2)

The [IPL1] session highlighted the difficulties people can have with communication and raised discussion about how to overcome this. (Respondent 58)

Students found the Dementia Friends session useful as it gave them a clearer understanding of how dementia affects people and the care they need:

[The face-to-face Dementia Friends session] was very helpful. Put it into perspective, and in a way I understood and has stayed with me. Gave me a better understanding and showed me how to explain it to people. (Respondent 1)

... the analogy used to describe dementia put it into an easy to understand way. One which can be used to discuss with people with dementia and their families. (Respondent 42)

The majority of students $(n=40)$ completed the face-to-face Dementia Friends session, which allowed students to discuss and share their different perspectives and experiences in interprofessional groups:

... personally, having experienced family dementia, the session was useful in that it opened up discussion with colleagues, and allowed me to understand their views on dementia. (Respondent 41) 
Students $(n=20)$ who had opted for the online option felt that it was good to become a

Dementia Friend in their own time:

... the online [option] allowed me to do it in my own time and refer back to when completing the workbook. (Respondent 27)

Students ( $n=19)$ mentioned that the video showed how dementia impacts on family members and the challenges they face:

... yes, it [the video] was very resourceful hearing from the family members as their experiences helped things make more sense. Helps build a picture. (Respondent 15)

... the video was helpful in hearing first-hand experiences from family members and friends who care for someone with dementia. This helped to see how carers cope and the challenges that they face. (Respondent 26)

A key message that emerged from the data was that students enjoyed the combination of face-to-face and online learning:

[Different learning methods] helped me to expand my knowledge of dementia, the signs and symptoms of the disease, and how to care best for those with dementia. A combination of sources of knowledge was useful as it kept the learning varied. (Respondent 14)

Most students ( $n=57)$ said that the structure of the reflective workbook was logical and easy to understand, allowing them to build on their knowledge in a structured way.

\section{Discussion}

This report describes the development, implementation and evaluation of pilot that aimed to provide a large number of healthcare students with a Tier 1 DA qualification by integrating it within an existing IPL opportunity. Amongst the 60 students who volunteered, all successfully 
completed the DA qualification and 57 students scored this learning experience as very helpful, or helpful, in developing their knowledge about dementia care. The two main themes emerging from the open-ended text comments emphasise the value of IPL and also to adopt a blended approach to optimise outcomes.

Students who participated in this initiative say they found it beneficial to learn together in small mixed professional groups during the two-hour, face-to-face IPL1 session since it helped them to understand the value of work together to care for these patients as professionals, as discussed by Balzer et al. (2016). This highlights the very core of IPL, its purpose and possibilities, as this insight is likely to cultivate future collaboration. Students enjoy the learning materiel being presented, both face-to-face and online, which resonate with that discussed by Riesen et al. (2012). According to these authors, a blended learning environment gives students flexibility and control over their learning, helping them to develop interprofessional competencies when learning with, from and about each other. More recently, Reeves and colleagues (2017) reveal in their scoping review that ease of access to online learning and the infusion of web-based technologies with more traditional approaches, indeed help strengthen interprofessional collaboration and networking.

The findings of this study should be considered in the light of a number of limitations. Firstly, the generalisability of this report is limited since it was conducted at a single institution. Secondly, the small sample size $(n=60)$ was voluntary and therefore inevitably subject to the self-selection biases of the participants. Thirdly, as discussed by Jackson et al. (2016), we acknowledge that findings presented here originate from a survey and that more rigorous 
research is needed to gain a deeper understanding of the longer-term impact of providing healthcare students with the Tier 1 Dementia Care qualification as part of an IPL exercise.

In conclusion, findings from this pilot gave this institution the confidence to roll this out to all IPL1 students since 2017-2018. Thus it has helped Health Education England and the UK government prepare our future workforce by giving them an opportunity to acquire a basic Dementia Awareness qualification, and grow the number of Dementia Friends in a learning environment where interprofessional collaboration is at the heart of their experience.

\section{Note}

1 Dementia Friends is a UK Alzheimer's Society's initiative that aims to transform the general public's awareness and actions about dementia. For further information see: https://www.dementiafriends.org.uk

\section{References}

Alushi, L., Hammond, J.A., \& Wood, J.H. (2015) Evaluation of dementia education programs for pre-registration healthcare students - A review of the literature. Nurse Education Today, 35, 992998. doi: 10.1016/j.nedt.2015.04.006

Alzheimer's Disease International (2016). World Alzheimer Report: Improving healthcare for people living with dementia. Retrieved https://www.alz.co.uk/research/WorldAlzheimerReport2016.pdf

Balzer, K., Schröder, R., Junghans, A., Stahl, U., Träder, Jens-Martin, \& Köpke, S. (2016) Improving competencies in evidence-based dementia care: Results from a pilot study on a novel interprofessional training course (the KOMPIDEM project). GMS Journal for Medical Education, 33(2), 1-22. doi: $10.3205 / \mathrm{zma001034}$

Cartwright, J., Franklin, D., Forman, D., Freegard, H. (2015) Promoting collaborative dementia care via online interprofessional education. Australasian Journal on Ageing, 34(2), 88-92. doi: 10.1111/ajag.12106 Health Education England. Tier 1 Learning Outcomes. Retrieved March 21, 2018, from https://hee.nhs.uk/our-work/dementia-awareness/resources-tier-one-two-three 
Jackson, M., Pelone, F., Reeves, S., Hassenkamp, A-M., Emery, C., Titmarsh, K., Greenwood, N. (2016) Interprofessional education in the care of people diagnosed with dementia and their carers: a systematic review BMJ Open, 6:e010948. doi:10.1136/bmjopen-2015010948

Reeves, S., Fletcher, S., McLoughlin, C., Yim, A. (2017) Interprofessional Online Learning for Primary Healthcare: findings from a scoping review. BMJ Open 2017:7:e016872. doi:10. 1136/bmjopen2017-016872

Riesen, E., Morley, M., Clendinneng, D., Ogilvie, S., \& Murray, M. A. (2012) Improving interprofessional competence in undergraduate students using a novel blended learning approach. Journal of Interprofessional Care 26(4), 312-318, doi: 10.3109/13561820.2012.660286 